\title{
EFEITOS DO AUMENTO DO TEOR DE FÓSFORO NA SEME NTE, OBTIDO VIA ADUBAÇÃO FOLIAR, NO CRESCIMENTO E NA NODULAÇÃO DO FEIJ OEIRO'(1)
}

\author{
A. P. ARAÚJ $\mathbf{O}^{(2)}$, M. G. TEIXEIRA ${ }^{(3)} \&$ E. R. LIMA(4)
}

\begin{abstract}
RESUMO
Sementes de feijoeiro (Phaseol us vulgaris L.) com alto teor de P, obtidas de plantas que receberam adubação foliar, foram utilizadas em um experimento em casa de vegetação para avaliar os efeitos do teor de $P$ na semente, no crescimento e na nodulação do feijoeiro no estádio vegetativo. $O$ experimento tinha arranjo fatorial $2 \times 2 \times 3 \times 3$ em blocos ao acaso com quatro repetições: dois cultivares (Carioca e R io Ti bagi), dois teores de P na semente (baixo e alto, correspondentes a 4,3 e 5,6 $\mathrm{mg} \mathrm{g}^{-1}$, em Carioca, e 4,8 e 6,3 $\mathrm{mg} \mathrm{g}^{-1}$, em Rio Tibagi), três doses de $P$ aplicado ao solo $\left(0,30\right.$ e $60 \mathrm{mg} \mathrm{kg}^{-1}$, respectivamente, $P_{0}, P_{30}$ e $\left.P_{60}\right)$ e três épocas de coleta (20, 30 e 40 dias após emergência - DAE ). 0 alto teor de $P$ na semente aumentou a matéria seca das plantas de feijoeiro em $P_{0}$ nas três coletas, e em $P_{30}$, aos 40 DAE. Plantas originadas de sementes com alto teor de $P$ mostraram menor dependência do suprimento de $P$ no solo, quanto à produção de matéria seca, do que aquelas oriundas de sementes com baixo P. $O$ alto teor de $P$ na semente aumentou o número de nódulos em Carioca em $P_{0}$, aos 20 e 30 DAE, e aumentou a massa de nódulos em $P_{30}$, aos 20 DAE nos dois cultivares e, aos 40 DAE, em $P_{30}$ em Carioca e em $P_{60}$ em Rio Tibagi. A acumulação de $\mathbf{N}$ na parte aérea aos 20 DAE foi superior nas plantas originadas de sementes com alto teor de $\mathbf{P}$. Segundo os resultados, sementes com alto teor de $\mathbf{P}$ obtido via adubação foliar podem aumentar o cresci mento, a nodulação e a acumulação de $\mathbf{N}$ do feijoeiro em estádios iniciais de crescimento, particularmente sob baixas doses de P aplicado ao solo.
\end{abstract}

Termos de indexação: feijão, nitrogênio, Phaseol us vulgaris, raiz.

(1) Apresentado na XXIV Reunião Brasileira de Fertilidade do Solo e N utrição de Plantas, em Santa Maria (RS), em outubro de 2000. Recebido para publicação em setembro de 2000 e aprovado em setembro de 2001.

(2) Professor do Departamento de Solos, Universidade Federal Rural do Rio de J aneiro - UFRRJ. CEP 23890-000 Seropédica (RJ). Bolsista do CNPq. E-mail: aparaujo@ufrrj.br

(3) Pesquisador da Embrapa Agrobiologia. CEP 23851-970 Seropédica (RJ). E-mail: grandi@cnpab.embrapa.br

(4) Mestrando em Fitotecnia, UFRRJ. Bolsista da CAPES. E-mail: elvis@rural net.com.br 


\title{
SUMMARY: EFFECTS OF INCREASED SEED PHOSPHORUS CONCEN- TRATION OBTAINED BY FOLIAR FERTILIZATION ON GROWTH AND NODULATION OF COMMON BEAN
}

\begin{abstract}
Seeds of common bean (Phaseolus vulgaris L.) with high P concentration, obtained from plants which had received foliar fertilization, wereused in a greenhouseexperiment to evaluate the effects of seed $\mathrm{P}$ concentration on growth and nodulation of common bean during the vegetativestage. The experiment had a $2 \times 2 \times 3 \times 3$ factorial randomized block design with four replicates: two cultivars (Carioca and RioT ibagi), two seed P concentrations (low and high, corresponding to 4.3 and $5.6 \mathrm{mg} \mathrm{g}^{-1}$ for Carioca, and 4.8 and $6.3 \mathrm{mg} \mathrm{g}^{-1}$ for Rio Tibagi), three levels of $\mathrm{P}$ applied to the soil ( 0,30 and $60 \mathrm{mg} \mathrm{kg}^{-1}$, respectively, $\mathrm{P}_{0}, \mathrm{P}_{30}$ and $\left.P_{60}\right)$ and three times of harvesting (20,30 and 40 days after emergence - DAE). The high seed $\mathrm{P}$ concentration increased the dry matter of bean plants at $\mathrm{P}_{0}$ at thethreeharvests, and at $P_{30}$ at 40 DAE. Plants originating from high $P$ seeds showed a lower dependence of soil $\mathrm{P}$ supply, in regard to dry matter production, than those originating from low $\mathrm{P}$ seeds. The high seed $\mathrm{P}$ concentration increased the number of nodules in Carioca at $\mathrm{P}_{0}$ by 20 and $30 \mathrm{DAE}$, and increased nodule mass at $P_{30}$ by $20 \mathrm{DAE}$ in both cultivars, and by 40 DAE at $P_{30}$ in Carioca and at $P_{60}$ in Rio Tibagi. The accumulation of $N$ by shoots at 20 DAE was greater in plants originating from high $P$ seeds. Theresults demonstratethat seeds with high $\mathrm{P}$ concentration obtained by foliar fertilization can enhance growth, nodulation and $\mathrm{N}$ accumulation of bean plants at early growth stages, particularly at low $\mathrm{P}$ levels applied to the soil.
\end{abstract}

Index terms: nitrogen, Phaseol us vulgaris, root.

\section{INTRODUÇÃO}

A deficiência de $P$ é generalizada nas regiões produtoras de feijão (Phaseolus vulgaris L.) no Brasil, e talvez este seja o fator nutricional mais limitante à fixação de $\mathrm{N}_{2}$ em pequenos cultivos. Como a fixação biológica de $\mathrm{N}_{2}$ é um processo de grande demanda energética, e como o $\mathrm{P}$ tem um papel relevante no metabolismo energético das células, a deficiência de $P$ tem um impacto negativo no estado energético dos nódul os (Sa \& I srael, 1991). Desta forma, oP teria um efeito benéfico na fixação de $\mathrm{N}_{2}$, tanto atuando diretamente sobre a iniciação, crescimento e funcionamento dos nódulos (I srael, 1987), aumentando sua atividade (Graham \& Rosas, 1979; Pereira \& Bliss, 1987), quanto estimulando o crescimento do hospedeiro (Gates \& Wilson, 1974).

Plantas de soja (Glycine max L. Merr.), quando dependentes da fixação biológica de $\mathrm{N}_{2}$, apresentaram maior requerimento de $\mathrm{P}$ para obtenção de crescimento ótimo do que plantas supridas com nitrato em solução nutritiva (I srael, 1987). Os parâmetros associados à fixação de $\mathrm{N}_{2}$ são mais dependentes do suprimento de $\mathrm{P}$ do que o próprio crescimento vegetal (Cassman et al., 1980; I srael, 1987). Em soja sob fixação simbiótica de $\mathrm{N}_{2}$, o estresse de P influiu no equilíbrio entrea biomassa de nódulos e de raízes de forma mais intensa que na partição de bi omassa entre raízes e parte aérea (Cassman et al., 1980). Além disto, os nódulos de feijoeiro constituem um forte dreno de $\mathrm{P}$, com grandes respostas às doses do nutriente (Graham \& Rosas, 1979).
O feijoei ro tem si do consi derado uma espécie com baixa fixação simbiótica de $\mathrm{N}_{2}$, quando comparadoa outras leguminosas de grão, em parte em virtude da susceptibilidade da espéciea estresses ambientais e nutricionais e ao curto período vegetativo (Piha \& Munns, 1987; Chaverra \& Graham, 1992).

Como o suprimento limitado de $\mathrm{P}$ causa atrasos no desenvolvimento da nodulação no feijoeiro (Araújo \& Teixeira, 2000), o maior teor de P em sementes defeijão poderia aumentar a disponibilidade do nutriente em estádios iniciais de infecção e formação dos nódulos, particularmente sob condições de baixa disponibilidade de P. Plantas de feijoeiro originadas de sementes com alta concentração de $P$ não só produziram maior massa seca de parteaérea e número e massa de nódulos, mas também mostraram-se menos dependentes do suprimento de $P$ no solo do que plantas oriundas de sementes com baixo teor de P (Teixeira et al., 1999). Altos teores de $P$ nas sementes aumentaram a massa seca de parte aérea, bem como o número e massa seca de nódulos de tremoço (Lupinus angustifolius L.) sob todas as doses de suprimento externo de $\mathrm{P}$, sobretudo sob deficiência de $P$ (Thomson et al., 1991).

A adubação foliar com $\mathrm{P}$ pode constituir uma alternativa tecnológica para elevar o teor de $\mathrm{P}$ nas sementes. Como sais fertilizantes podem ser rapidamente absorvidos e metabolizados pelas folhas, é possível aplicar nutrientes em cultivos em estádios de alta demanda de nutrientes, como durante o rápido crescimento vegetativo e o desenvol vimento dos frutos (Sesay \& Shibles, 1980). 
A adubação foliar com solução com $10 \mathrm{~g} \mathrm{~L}^{-1}$ de $P$ aumentou o teor de $P$ nas sementes de feijoeiro, eas aplicações no estádio de formação das vagens mostraram-se mais efetivas no aumento do teor de P nas sementes (Teixeira \& Araújo, 1999).

Estetrabal ho teve como objetivo avaliar os efeitos do maior teor deP nas sementes, obti do via adubação foliar, no crescimento e nodulação do feijoeiro no estádio vegetativo.

\section{MATERIAL E MÉTODOS}

Foi realizado um experimento em casa de vegetação na E mbrapa Agrobi ol ogia, em Seropédica (RJ ), no delineamento blocos ao acaso com quatro repetições, em esquema fatorial $2 \times 2 \times 3 \times 3$ : dois cultivares de feijoeiro (Carioca e Rio Tibagi), dois níveis de $\mathrm{P}$ na semente (baixo e alto), três doses deP aplicadas ao solo $\left(0,30 \mathrm{e}^{\circ} \mathrm{mg} \mathrm{kg}^{-1}\right.$, respectivamente, $P_{0}, P_{30}$ e $P_{60}$ ) e três épocas de coleta (aos 20, 30 e 40 dias após emergência - DAE). Foram utilizadas as sementes obtidas por Teixeira \& Araújo (1999) em condições de campo, sendo as sementes com alto teor de $\mathrm{P}$ colhidas de parcelas que receberam adubação foliar com $10 \mathrm{~g} \mathrm{P} \mathrm{L}^{-1}$ aos 45 e 60 dias após emergência, e as sementes com baixo teor de $\mathrm{P}$ col hidas de parcelas sem adubação foliar com P. As características das sementes utilizadas estão no quadro 1. Cabe ressaltar que Teixeira \& Araújo (1999) buscaram reproduzir as condições usuais em um cultivo para produção de sementes, aplicando no plantio $40 \mathrm{~kg} \mathrm{ha}^{-1}$ de $\mathrm{P}$, o que resultou em sementes com adequado teor de $\mathrm{P}$ mesmo nas parcelas que não receberam adubação foliar (Quadro 1).

Utilizou-se como substrato o horizonte A de um Argissolo, passado em peneira de $6 \mathrm{~mm}$ e colocado em vasos de $3 \mathrm{~kg}$. As análises do solo (EMBRAPA, 1997) apresentaram: $\mathrm{pH}$ em água $4,8,5 \mathrm{mmol}_{\mathrm{c}} \mathrm{dm}^{-3}$ de $\mathrm{Al}, 11 \mathrm{mmol}_{\mathrm{C}} \mathrm{dm}^{-3}$ de $\mathrm{Ca}, 12 \mathrm{mmol}_{\mathrm{C}} \mathrm{dm}^{-3}$ de $\mathrm{Mg}$, $1,3 \mathrm{mmol}_{\mathrm{c}} \mathrm{dm}^{-3}$ de $\mathrm{K}, 3 \mathrm{mg} \mathrm{dm}^{-3} \mathrm{deP}$. O sol o decada vaso recebeu $1,5 \mathrm{~g}$ de $\mathrm{CaCO}_{3}$ e, 10 dias após, adubação na forma de produtos p.a., diluídos em

Quadro 1. Características das sementes utilizadas

\begin{tabular}{lccc}
\hline Cultivar & $\begin{array}{c}\text { Nível de P na } \\
\text { semente }\end{array}$ & $\begin{array}{c}\text { Peso de } \\
\text { 100 sementes }\end{array}$ & Teor de P \\
\hline \multirow{2}{*}{ Carioca } & Baixo & 19,4 & $\mathrm{mg} \mathrm{g}^{-1}$ \\
& Alto & 19,0 & 4,3 \\
Rio Tibagi & Baixo & 13,6 & 5,6 \\
& Alto & 14,8 & 4,8 \\
& & & 6,3 \\
\hline
\end{tabular}

água; posteriormente, o material de cada vaso foi homogeneizado. As seguintes doses foram aplicadas (em mg kg${ }^{-1}$ de solo): $10 \mathrm{Mg}$ (como $\mathrm{MgSO}_{4} \cdot 7 \mathrm{H}_{2} \mathrm{O}$ ), $2 \mathrm{Cu}\left(\mathrm{comoCuSO}_{4} \cdot 5 \mathrm{H}_{2} \mathrm{O}\right), 1 \mathrm{Zn}\left(\mathrm{comoZnSO}_{4} \cdot 7 \mathrm{H}_{2} \mathrm{O}\right)$, $0,05 \mathrm{~B}\left(\mathrm{comoH}_{3} \mathrm{BO}_{3}\right), 0,2 \mathrm{Mo}\left(\mathrm{como} \mathrm{Na} \mathrm{M} \mathrm{OO}_{4} \cdot 2 \mathrm{H}_{2} \mathrm{O}\right)$, $1 \mathrm{Fe}$ (como Fe-EDTA), 0, 30 e $60 \mathrm{P}\left(\right.$ como $\mathrm{KH}_{2} \mathrm{PO}_{4}$ ), nos diferentes tratamentos com P. Nas doses mais baixas de $\mathrm{P}$, o $\mathrm{K}$ foi complementado com $\mathrm{KCl}$ para homogeneizar a dose de K. A semeadura foi efetuada sete dias após a adubação, sendo as sementes inoculadas com as estirpes BR266, BR281 e BR322 de Rhizobium spp., da coleção da Embrapa Agrobiologia. Foram cultivadas quatro plantas por vaso após desbaste.

Nas coletas, os caules e fol has foram separados, determinando-se a área foliar (Li-Cor 3100 Area Meter). As raízes foram lavadas sobre uma peneira, e os nódulos foram separados e contados. As fol has, caules, raízes e nódul os foram secos em estufa a $70^{\circ} \mathrm{C}$ e pesados. Na parte aérea (fol has + caules), foram determinadas: a concentração de $\mathrm{P}$ após digestão nitro-perclórica, e dosagem colorimétrica pelo molibdato de amônio, e a concentração de $\mathrm{N}$ pelo método semimicro Kjeldahl (Malavolta et al., 1989).

A homogeneidade das variâncias dos dados obtidos nas três col etas foi verificada pelo teste de Hartley (Neter et al., 1990). Por ter a maioria dos caracteres apresentado variâncias heterocedásticas, indusive após transformação em logaritmo natural, a análise de variância foi efetuada para cada coleta separadamente, avaliando os efeitos dos fatores (cultivar, P do sol o eP da semente) e suas interações.

\section{RESULTADOS E DISCUSSÃO}

De forma geral, os cultivares Carioca e RioTibagi responderam de forma similar aos aumentos no teor de P na sementee nas doses de P no solo. O cultivar Carioca produziu maior área foliar, massa seca de folha e de caule enúmero de nódul os que RioTibagi, nas três épocas avaliadas (Quadro 2). Por outrolado, RioTibagi apresentou maior massa de raiz e razão raiz:parte aérea que Carioca nas três coletas, confirmando seu potencial de produção de biomassa radicular (Araújo et al., 2000).

Aos 20 DAE, não houve aumento significativo da área fol iar e do peso de matéria seca de fol ha e total entre as doses $P_{30}$ e $P_{60}$, de forma diferente do observado aos 30 e 40 DAE (Quadro 3). I sto significa que o suprimento adicional de $\mathrm{P}$ no solo, na dose $\mathrm{P}_{60}$, só foi convertido em biomassa da parte aérea após os $20 \mathrm{DAE}$. Em geral, não houve aumento significativo da massa seca de raiz e de caule entre as doses $P_{30}$ e $P_{60}$ nas três coletas (Quadro 3), indicando que as plantas de feijoeiro utilizaram o suprimento adicional de $\mathrm{P}$ do solo, na dose $\mathrm{P}_{60}$, preferencialmente para a produção de fol has. 
Quadro 2. Área foliar, peso de matéria seca de diferentes porções vegetais, número de nódulos e razão raiz:parte aérea de dois cultivares de feijoei ro em três épocas de coleta (médias de dois níveis de $P$ na semente e de três doses de $P$ no solo)

\begin{tabular}{|c|c|c|c|c|c|c|c|c|c|c|c|c|}
\hline \multirow{3}{*}{ Cultivar } & & & & \multicolumn{9}{|c|}{ Matéria seca } \\
\hline & \multicolumn{3}{|c|}{ Área foliar } & \multicolumn{3}{|c|}{ Folha } & \multicolumn{3}{|c|}{ Caule } & \multicolumn{3}{|c|}{ Raiz } \\
\hline & 20 DAE & 30 DAE & 40 DAE & 20 DAE & 30 DAE & 40 DAE & 20 DAE & 30 DAE & 40 DAE & 20 DAE & 30 DAE & $40 \mathrm{DAE}$ \\
\hline & $-\mathrm{Cl}$ & $n^{2}$ planta & -1 & & & 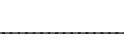 & - $n$ & ig planta & & & & \\
\hline Carioca & $161 \mathrm{a}$ & $193 \mathrm{a}$ & 229 a & 571 a & 844 a & 908 a & 176 a & $263 \mathrm{a}$ & 391 a & $347 \mathrm{~b}$ & $344 \mathrm{~b}$ & $387 \mathrm{~b}$ \\
\hline Rio Tibagi & $131 \mathrm{~b}$ & $151 \mathrm{~b}$ & $186 \mathrm{~b}$ & $482 \mathrm{~b}$ & $686 \mathrm{~b}$ & $819 \mathrm{~b}$ & $142 \mathrm{~b}$ & $201 \mathrm{~b}$ & 296 b & $380 a$ & 377 a & $476 a$ \\
\hline
\end{tabular}

\begin{tabular}{|c|c|c|c|c|c|c|c|c|c|c|c|}
\hline \multicolumn{3}{|c|}{$\begin{array}{c}\text { Matéria seca de } \\
\text { nódulos }\end{array}$} & \multicolumn{3}{|c|}{ Matéria seca total } & \multicolumn{3}{|c|}{ Número de nódulos } & \multicolumn{3}{|c|}{ Razão raiz:parte aérea } \\
\hline 20 DAE & 30 DAE & 40 DAE & 20 DAE & 30 DAE & 40 DAE & 20 DAE & 30 DAE & 40 DAE & 20 DAE & 30 DAE & 40 DAE \\
\hline & & $\mathrm{mg} \mathrm{pl}$ & anta-1 & & & $\longrightarrow$ & $n^{\circ}$ planta & -1 & $\longrightarrow$ & $-\mathrm{mg} \mathrm{g}^{-1}$ & \\
\hline $43 \mathrm{a}$ & $65 a$ & $62 a$ & 1126 a & 1516 a & 1747 a & 77 a & $111 \mathrm{a}$ & $141 \mathrm{a}$ & $531 \mathrm{~b}$ & $383 \mathrm{~b}$ & $383 \mathrm{~b}$ \\
\hline $36 \mathrm{~b}$ & 59 a & $63 a$ & $1039 \mathrm{~b}$ & $1323 \mathrm{~b}$ & $1653 \mathrm{~b}$ & $44 \mathrm{~b}$ & $83 \mathrm{~b}$ & $105 \mathrm{~b}$ & $685 a$ & $546 a$ & $550 \mathrm{a}$ \\
\hline
\end{tabular}

DAE: dias após emergência. Médias seguidas por letras diferentes, dentro da mesma coluna, diferem entre si pelo teste Tukey a 5\%.

Quadro 3. Área foliar, peso de matéria seca de diferentes porções vegetais e razão raiz:parte aérea de de plantas de feijoeiro em três épocas de coleta, originadas de sementes com baixo e alto teor de $P$, e crescidas sob três doses de P no solo (médias de dois cultivares)

\begin{tabular}{|c|c|c|c|c|c|c|c|}
\hline \multirow{2}{*}{ Coleta } & \multirow{2}{*}{ Dose de $P$} & \multicolumn{2}{|c|}{ Área foliar } & \multicolumn{2}{|c|}{ Matéria seca de folha } & \multicolumn{2}{|c|}{ Matéria seca de caule } \\
\hline & & Baixo P & Alto P & Baixo P & Alto P & Baixo P & Alto P \\
\hline & $\mathrm{mg} \mathrm{kg}^{-1}$ & \multicolumn{2}{|c|}{$\mathrm{cm}^{2} \mathrm{planta}^{-1} \ldots$} & \multicolumn{4}{|c|}{ mg planta-1 $^{-1}$} \\
\hline $20 \mathrm{DAE}$ & $\begin{array}{r}0 \\
30 \\
60\end{array}$ & $\begin{array}{l}106 \mathrm{~b} \\
152 \mathrm{a} \\
160 \mathrm{a}\end{array}$ & $\begin{array}{l}129 b^{*} \\
166 a^{*} \\
162 a\end{array}$ & $\begin{array}{l}366 \mathrm{~b} \\
552 \mathrm{a} \\
563 \mathrm{a}\end{array}$ & $\begin{array}{l}479 b^{*} \\
623 a^{*} \\
576 a\end{array}$ & $\begin{array}{l}123 \mathrm{~b} \\
161 \mathrm{a} \\
156 \mathrm{a}\end{array}$ & $\begin{array}{l}142 b^{*} \\
183 a^{*} \\
159 b\end{array}$ \\
\hline $30 \mathrm{DAE}$ & $\begin{array}{r}0 \\
30 \\
60\end{array}$ & $\begin{array}{r}67 c \\
196 b \\
235 a\end{array}$ & $\begin{array}{l}94 c^{*} \\
207 b \\
232 a\end{array}$ & $\begin{array}{r}310 \mathrm{c} \\
860 \mathrm{~b} \\
1016 \mathrm{a}\end{array}$ & $\begin{array}{r}452 b^{*} \\
928 \text { a } \\
1024 a\end{array}$ & $\begin{array}{l}140 \mathrm{~b} \\
250 \mathrm{a} \\
272 \mathrm{a}\end{array}$ & $\begin{array}{l}185 b^{*} \\
270 a \\
278 a\end{array}$ \\
\hline \multirow[t]{4}{*}{$40 \mathrm{DAE}$} & $\begin{array}{r}0 \\
30 \\
60\end{array}$ & $\begin{array}{r}73 c \\
229 b \\
301 a\end{array}$ & $\begin{array}{r}89 c \\
245 b \\
307 a\end{array}$ & $\begin{array}{r}300 \mathrm{c} \\
880 \mathrm{~b} \\
1207 \mathrm{a}\end{array}$ & $\begin{array}{r}398 c^{*} \\
1112 b^{*} \\
1283 a\end{array}$ & $\begin{array}{l}175 c \\
366 b \\
432 a\end{array}$ & $\begin{array}{l}227 b^{*} \\
427 a^{*} \\
432 a\end{array}$ \\
\hline & & \multicolumn{2}{|c|}{ Matéria seca de raiz } & \multicolumn{2}{|c|}{ Matéria seca total } & \multicolumn{2}{|c|}{ Razão raiz:parte aérea } \\
\hline & & Baixo P & Alto P & Baixo P & Alto P & Baixo P & Alto P \\
\hline & & \multicolumn{4}{|c|}{ mg planta-1 } & \multicolumn{2}{|c|}{$\ldots \mathrm{mg} \mathrm{g}^{-1}$} \\
\hline $20 \mathrm{DAE}$ & $\begin{array}{r}0 \\
30 \\
60\end{array}$ & $\begin{array}{l}301 \mathrm{~b} \\
432 \mathrm{a} \\
400 \mathrm{a}\end{array}$ & $\begin{array}{l}346 \text { a } \\
367 \text { a* } \\
337 \text { a* }\end{array}$ & $\begin{array}{r}792 \text { b } \\
1175 \text { a } \\
1190 \mathrm{a}\end{array}$ & $\begin{array}{l}972 b^{*} \\
1215 \text { a } \\
1153 \text { a }\end{array}$ & $\begin{array}{l}638 a \\
665 a \\
670 a\end{array}$ & $\begin{array}{l}592 \text { a } \\
514 \text { a* } \\
569 \text { a* }\end{array}$ \\
\hline $30 \mathrm{DAE}$ & $\begin{array}{r}0 \\
30 \\
60\end{array}$ & $\begin{array}{l}247 b \\
407 a \\
391 a\end{array}$ & $\begin{array}{l}309 c^{*} \\
433 a \\
376 b\end{array}$ & $\begin{array}{r}698 \mathrm{c} \\
1586 \mathrm{~b} \\
1799 \mathrm{a}\end{array}$ & $\begin{array}{l}954 b^{*} \\
1695 a \\
1784 \text { a }\end{array}$ & $\begin{array}{l}596 a \\
445 b \\
399 b\end{array}$ & $\begin{array}{l}529 a \\
441 b \\
376 b\end{array}$ \\
\hline $40 \mathrm{DAE}$ & $\begin{array}{r}0 \\
30 \\
60\end{array}$ & $\begin{array}{l}294 \mathrm{~b} \\
487 \mathrm{a} \\
491 \mathrm{a}\end{array}$ & $\begin{array}{l}345 b \\
523 a \\
449 a\end{array}$ & $\begin{array}{r}772 \text { c } \\
1796 \text { b } \\
2239 \text { a }\end{array}$ & $\begin{array}{r}974 b^{*} \\
2136 a^{*} \\
2283 a\end{array}$ & $\begin{array}{l}630 a \\
468 b \\
375 b\end{array}$ & $\begin{array}{l}591 a \\
398 b^{*} \\
336 b\end{array}$ \\
\hline
\end{tabular}

$\overline{\mathrm{DAE}}$ : dias após emergência. Médias seguidas por letras diferentes, dentro da mesma col una e da mesma col eta, diferem entre si pelo teste Tukey a 5\%. * Diferença significativa entre teor de $\mathrm{P}$ na semente pelo teste Tukey a $5 \%$. 
$O$ altoteor de $\mathrm{P}$ na semente estimulou a expansão foliar do feijoeiro, com aumentos na área foliar e na matéria seca de fol ha em $\mathrm{P}_{0}$ e $\mathrm{P}_{30}$, aos $20 \mathrm{DAE}$, e em $\mathrm{P}_{0}$, aos $30 \mathrm{DAE}$ (Quadro 3). Aos $40 \mathrm{DAE}$, o al to teor de $P$ na semente aumentou a matéria seca de fol ha $\mathrm{emP}_{0} \mathrm{eP}_{30}$, sem, no entanto, influir significativamente na área fol iar. A acumulação de bi omassa apresentou comportamento similar: o alto teor de P na semente aumentou a matéria seca de caule etotal em $\mathrm{P}_{0}$, nas três col etas, e em $\mathrm{P}_{30}$, aos 40 DAE (Quadro 3).

Estes resultados demonstram que o maior teor deP na semente estimulou o crescimento vegetativo do feijoeiro, mas apenas nas doses mais baixas de $P$ aplicadas ao solo. Resultados com trigo (Triticum aestivum L.) (Bolland \& Baker, 1988; De Marco, 1990), tremoço (Thomson et al., 1991), leguminosas forrageiras (Bolland \& Paynter, 1990) e feijoeiro (Teixeira et al., 1999) também fornecem evidências de que os efeitos do maior teor de $\mathrm{P}$ da semente no estímulo ao crescimento da parte aérea diminuem com a maior disponibilidade deP no solo. Estemaior crescimento de plantas de feijoeiro, acarretado pelas sementes com alto teor de $\mathrm{P}$, manteve-se durante todo o estádio vegetativo, como observado em tremoço (Thomson et al., 1991).

O maior crescimento inicial do feijoeiro, oriundo de sementes com al to teor de $\mathrm{P}$, pode assumir impactos positivos na produção de grãos, particularmentesob condições de estresseambiental . Sementes de feijão produzidas sob altas doses de fertilizante propiciaram maior número de plantas em condições adversas à germinação (Vieira, 1986). Sementes detrigo obtidas de sítios com aplicação de $\mathrm{P}$ garantiram mel hor estabelecimento das plântulas e mai or produção de matéria seca, que se refletiram no incremento do rendimento de grãos, mas apenas sob condições de cultivo na época seca (Burnett et al., 1997).

Nas plantas oriundas de sementes com baixo teor de $\mathrm{P}$, houve aumentos significativos na matéria seca total da dose $P_{30}$ para $P_{60}$ aos 30 e 40 DAE; já nas plantas originadas de sementes com alto teor de $P$, estes aumentos não foram significativos (Quadro 3). Isto indica que plantas de feijoeiro originadas de sementes com al to teor de $\mathrm{P}$ são menos dependentes do suprimento de $P$ no solo para produção de biomassa do que plantas oriundas de sementes com baixo P, confirmando observações de Teixeira et al. (1999). Sementes de trevo subterrâneo (Trifolium subterraneum L.) com al ta concentração de P reduziram a resposta ao $\mathrm{P}$ aplicado ao solo, modificando o requerimento para a obtenção do crescimento máximo (Paynter, 1993).

As respostas do crescimento radicular ao aumento do teor de $\mathrm{P}$ na semente foram mais complexas. Aos $20 \mathrm{DAE}$, o alto teor de $\mathrm{P}$ na semente diminuiu a matéria seca de raiz em $P_{30}$ e $P_{60}$ (Quadro 3), em comparação ao baixo teor de $\mathrm{P}$ na semente, indicando que, sob condições de adequado suprimento de $\mathrm{P}$ no solo, o fornecimento adicional deP da sementefoi utilizado preferencialmente para produção de biomassa de parte aérea. I sto causou uma redução concomitante da razão raiz:parte aérea nas plantas oriundas de sementes com alto teor de P (Quadro 3). J á aos $30 \mathrm{DAE}$, o maior teor de P na semente aumentou o crescimento radicular em $\mathrm{P}_{0}$ (Quadro 3), ou seja, sob deficiência deP, o suprimento adicional de $P$ da semente foi utilizado no crescimento radicular. Em trigo, o crescimento radicular também foi estimulado pelo alto teor de $\mathrm{P}$ da semente, mas em menor extensão que o crescimento da parte aérea, o que reduziu a razão raiz:parteaérea em plantas originadas de sementes com alto teor de P (De Marco, 1990).

De formal geral, o número e o peso de nódulos aumentaram com o incremento das doses de $\mathrm{P}$ no sol o nas três épocas avaliadas (Quadro 4). Enquanto a matéria seca total das plantas de feijoeiro aumentou em média 2,6 vezes entre as doses $P_{0} e$ $\mathrm{P}_{60}$ aos 40 DAE (Quadro 3), o peso de nódulos aumentou mais de 30 vezes (Quadro 4), confirmando que os caracteres associados à nodul ação mostraramse mais dependentes do suprimento de $\mathrm{P}$ do que o crescimento do hospedei ro (I srael, 1987).

O altoteor deP da sementeaumentou significativamente o número de nódulos do cultivar Carioca na dose $P_{0}$, aos 20 e 30 DAE; já em Rio Tibagi, o $P$ da semente não influenciou o número de nódulos (Quadro 4). O al to teor de P da semente aumentou a matéria seca de nódulos em $P_{30}$, aos 20 DAE nos dois cultivares, em $P_{30}$, aos $40 \mathrm{DAE}$, em Carioca, e em $\mathrm{P}_{60}$ aos $40 \mathrm{DAE}$, em Rio Tibagi (Quadro 4). Na menor dose de $\mathrm{P}$ no solo, a nodulação foi bastante limitada, e o suprimento adicional de P da semente foi utilizado preferencialmente no crescimento do hospedeiro, com aumento na biomassa total das plantas oriundas de sementes com alto teor de $\mathrm{P}$ (Quadro 3). J á sob maior disponibilidade de $\mathrm{P}$ no sol o, o suprimento adicional de P das sementes com alto teor de $\mathrm{P}$ pôde ser destinado ao crescimento de nódulos, com aumentos da massa de nódul os em $P_{30}$ e $\mathrm{P}_{60}$ (Quadro 4). $\mathrm{O}$ alto teor de $\mathrm{P}$ da semente aumentou o peso de cada nódulo aos 20 e $30 \mathrm{DAE}$ (dados não apresentados), ou seja, sementes com al to teor de P estimularam a nodulação do feijoeiro através principal mente do aumento do tamanho do nódulo.

Os efeitos do aumento do teor de $\mathrm{P}$ da semente na nodulação do feijoeiro não foram tão pronunciados quanto os registrados por Teixeira et al. (1999). Entretanto, estes autores trabal haram com sementes com uma mais ampla variação da concentração de $\mathrm{P}$, obti das pela modificação das doses de P aplicadas a um sol o de baixa fertilidade natural. A menor amplitude de variação na concentração de $P$ das sementes utilizadas no atual experimento (Quadro 1) pode ter reduzido os efeitos do $\mathrm{P}$ da semente na nodulação do feijoeiro. 
O cultivar Carioca acumul ou mais $\mathrm{N}$ eP na parte aérea que Rio Tibagi nas três épocas de coleta, em virtude principalmente de seu maior crescimento, pois os teores de $\mathrm{N}$ e de $\mathrm{P}$ foram similares nos dois cultivares (Quadro 5). Considerando a média dos cultivares e doses de $\mathrm{P}$ no solo, $\mathrm{O}$ alto teor de $\mathrm{P}$ da semente aumentou o teor de $\mathrm{N}$ na parte aérea aos 20 DAE (Quadro 5). Esta maior acumulação de N foi atribuída, principalmente, ao maior crescimento das plantas originadas de sementes com alto teor de $\mathrm{P}$, já que o teor de $\mathrm{N}$ na parte aérea não foi modificado neste estádio (Quadro 5). O alto teor de $\mathrm{P}$ da semente causou redução dos teores de $\mathrm{N}$ e de $\mathrm{P}$ da parte aérea aos $40 \mathrm{DAE}$, em virtude de efeitos de diluição causados por um estímulo ao crescimento da parte aérea verificados neste estádio, sem concomitantes aumentos da acumulação destes nutrientes (Quadros 3 e 5). Em leguminosas forrageiras, 0 teor de $P$ na parte aérea não foi alterado pela concentração de $P$ nas sementes, mas o teor de $\mathrm{P}$ aumentou em conseqüência da maior produção de biomassa (Bolland \& Paynter, 1990).

O suprimento adicional das sementes com alto teor de $\mathrm{P}$ foi de $0,23 \mathrm{mg}$ de $\mathrm{P}$ por planta, na média dos dois cultivares (Quadro 1 ), valor superior ao aumento do teor de $\mathrm{P}$ na parte aérea das plantas oriundas de sementes com alto teor de P (Quadro 5). Sendo assim, o estímulo do al to teor deP da semente

Quadro 4. Número e peso de matéria seca de nódulos de dois cultivares de feijoeiro em três épocas de coleta, originados de sementes com baixo e alto teor de P e crescidos sob três doses de P no solo

\begin{tabular}{|c|c|c|c|c|c|c|c|c|c|}
\hline \multirow{3}{*}{ Coleta } & \multirow{3}{*}{$\begin{array}{c}\text { Dose de P } \\
\text { no solo }\end{array}$} & \multicolumn{4}{|c|}{ Número de nódulos } & \multicolumn{4}{|c|}{ Matéria seca de nódulos } \\
\hline & & \multicolumn{2}{|c|}{ Carioca } & \multicolumn{2}{|c|}{ Rio Tibagi } & \multicolumn{2}{|c|}{ Carioca } & \multicolumn{2}{|c|}{ Rio Tibagi } \\
\hline & & Baixo P & Alto P & Baixo P & Alto P & Baixo P & Alto P & Baixo P & Alto P \\
\hline & $\mathrm{mg} \mathrm{kg}^{-1}$ & $\longrightarrow$ & $n^{\circ} \mathrm{p}$ & $\mathrm{nta}^{-1}$ & - & $\longrightarrow$ & - $\mathrm{mg} \mathrm{r}$ & inta-1 & - \\
\hline $20 \mathrm{DAE}$ & $\begin{array}{r}0 \\
30 \\
60\end{array}$ & $\begin{array}{r}23 c \\
83 b \\
114 a\end{array}$ & $\begin{array}{r}44 b^{*} \\
88 \mathrm{a} \\
112 \mathrm{a}\end{array}$ & $\begin{array}{l}15 \mathrm{~b} \\
45 \mathrm{a} \\
66 \mathrm{a}\end{array}$ & $\begin{array}{l}23 \mathrm{~b} \\
55 \mathrm{a} \\
58 \mathrm{a}\end{array}$ & $\begin{array}{r}3 c \\
36 b \\
78 a\end{array}$ & $\begin{array}{c}6 \mathrm{c} \\
48 b^{*} \\
87 \mathrm{a}\end{array}$ & $\begin{array}{r}3 c \\
26 b \\
68 a\end{array}$ & $\begin{array}{c}5 \mathrm{c} \\
39 \mathrm{~b}^{*} \\
75 \mathrm{a}\end{array}$ \\
\hline $30 \mathrm{DAE}$ & $\begin{array}{r}0 \\
30 \\
60\end{array}$ & $\begin{array}{r}24 \mathrm{c} \\
129 \mathrm{~b} \\
174 \mathrm{a}\end{array}$ & $\begin{array}{l}52 \mathrm{c}^{*} \\
128 \mathrm{~b} \\
160 \mathrm{a}\end{array}$ & $\begin{array}{r}13 \mathrm{~b} \\
108 \mathrm{a} \\
133 \mathrm{a}\end{array}$ & $\begin{array}{r}25 c \\
83 b \\
137 a\end{array}$ & $\begin{array}{r}3 \mathrm{c} \\
70 \mathrm{~b} \\
121 \mathrm{a}\end{array}$ & $\begin{array}{r}10 \mathrm{c} \\
70 \mathrm{~b} \\
108 \mathrm{a}\end{array}$ & $\begin{array}{r}2 c \\
68 \mathrm{~b} \\
110 \mathrm{a}\end{array}$ & $\begin{array}{r}7 \mathrm{c} \\
59 \mathrm{~b} \\
108 \mathrm{a}\end{array}$ \\
\hline $40 \mathrm{DAE}$ & $\begin{array}{r}0 \\
30 \\
60\end{array}$ & $\begin{array}{r}30 c \\
139 b \\
229 a\end{array}$ & $\begin{array}{r}58 \mathrm{c} \\
158 \mathrm{~b} \\
230 \mathrm{a}\end{array}$ & $\begin{array}{r}12 \mathrm{~b} \\
141 \mathrm{a} \\
178 \mathrm{a}\end{array}$ & $\begin{array}{r}18 c \\
118 b \\
163 a\end{array}$ & $\begin{array}{r}4 c \\
54 b \\
118 a\end{array}$ & $\begin{array}{c}9 \mathrm{~b} \\
81 \mathrm{a}^{*} \\
109 \mathrm{a}\end{array}$ & $\begin{array}{r}2 c \\
73 b \\
102 a\end{array}$ & $\begin{array}{r}3 c \\
69 \mathrm{~b} \\
127 \mathrm{a} *\end{array}$ \\
\hline
\end{tabular}

DAE : dias após emergência. Médias seguidas por letras diferentes, dentro da mesma coluna e da mesma col eta, diferem entre si pelo testeTukey a 5\%. * Diferença significativa entre teor de P na semente pelo teste Tukey a 5\%.

Quadro 5. Teores e conteúdos de N e de P na parte aérea de dois cultivares de feijoei ro em três épocas de coleta, originados de sementes com baixo e alto teor de P e crescidos sob três doses de P no solo

\begin{tabular}{|c|c|c|c|c|c|c|c|c|c|c|c|c|}
\hline \multirow{2}{*}{ Tratamento } & \multicolumn{3}{|c|}{ Teor de N na parte aérea } & \multicolumn{3}{|c|}{ Teor de $\mathrm{N}$ na parte aérea } & \multicolumn{3}{|c|}{ Teor de $\mathrm{P}$ na parte aérea } & \multicolumn{3}{|c|}{ Teor de $\mathrm{P}$ na parte aérea } \\
\hline & $20 \mathrm{DAE}$ & $30 \mathrm{DAE}$ & $40 \mathrm{DAE}$ & $20 \mathrm{DAE}$ & $30 \mathrm{DAE}$ & $40 \mathrm{DAE}$ & $20 \mathrm{DAE}$ & $30 \mathrm{DAE}$ & $40 \mathrm{DAE}$ & $20 \mathrm{DAE}$ & $30 \mathrm{DAE}$ & $40 \mathrm{DAE}$ \\
\hline Cultivar & \multicolumn{3}{|c|}{$\mathrm{mg} \mathrm{g}^{-1}$} & \multicolumn{3}{|c|}{$\mathrm{mg} \mathrm{planta}^{-1}$} & \multicolumn{3}{|c|}{$\mathrm{mg} \mathrm{g}^{-1}$} & \multicolumn{3}{|c|}{ mg planta $^{-1}$} \\
\hline Carioca & 31,7 a & $28,0 \mathrm{a}$ & 27,6 a & 23,6 a & 31,8 a & $37,7 a$ & $1,59 \mathrm{a}$ & $1,47 \mathrm{~b}$ & $1,62 \mathrm{a}$ & $1,20 \mathrm{a}$ & $1,75 \mathrm{a}$ & $2,28 \mathrm{a}$ \\
\hline Rio Tibagi & $29,5 b$ & $26,0 \mathrm{~b}$ & 27,8 a & $18,3 \mathrm{~b}$ & $22,9 \mathrm{~b}$ & $30,9 \mathrm{~b}$ & $1,64 \mathrm{a}$ & $1,60 \mathrm{a}$ & $1,73 \mathrm{a}$ & $1,07 \mathrm{~b}$ & $1,54 \mathrm{~b}$ & $2,04 \mathrm{~b}$ \\
\hline \multicolumn{13}{|c|}{ Dose de P no solo (mg kg-1) } \\
\hline 0 & 28,6 b & $25,7 \mathrm{~b}$ & $25,1 \mathrm{~b}$ & $15,7 \mathrm{c}$ & $13,7 \mathrm{c}$ & $13,4 \mathrm{c}$ & $0,87 \mathrm{c}$ & $0,99 c$ & $1,16 \mathrm{c}$ & $0,47 c$ & $0,51 \mathrm{c}$ & $0,62 \mathrm{c}$ \\
\hline 30 & $28,3 b$ & $24,3 b$ & $27,0 \mathrm{~b}$ & $21,6 b$ & $27,9 \mathrm{~b}$ & $37,4 \mathrm{~b}$ & $1,50 \mathrm{~b}$ & $1,48 b$ & $1,75 \mathrm{~b}$ & $1,12 \mathrm{~b}$ & $1,67 \mathrm{~b}$ & $2,37 b$ \\
\hline 60 & $34,9 a$ & 31,0 a & 31,0 a & 25,5 a & $40,4 \mathrm{a}$ & 52,1 a & $2,49 a$ & $2,13 \mathrm{a}$ & $2,10 a$ & $1,81 \mathrm{a}$ & $2,74 \mathrm{a}$ & $3,49 a$ \\
\hline \multicolumn{13}{|c|}{ Teor de $P$ na semente } \\
\hline Baixo P & 30,6 a & 27,4 a & 29,5 a & $19,7 \mathrm{~b}$ & 26,6 a & 33,7 a & $1,66 \mathrm{a}$ & $1,57 \mathrm{a}$ & $1,79 \mathrm{a}$ & $1,11 \mathrm{a}$ & $1,63 \mathrm{a}$ & $2,15 a$ \\
\hline Alto P & 30,6 a & 26,6 a & $26,0 \mathrm{~b}$ & $22,2 \mathrm{a}$ & 28,1 a & 34,9 a & $1,58 \mathrm{a}$ & $1,50 \mathrm{a}$ & $1,56 b$ & $1,16 \mathrm{a}$ & $1,66 \mathrm{a}$ & $2,18 \mathrm{a}$ \\
\hline
\end{tabular}

DAE : dias após emergência. Médias seguidas por letras diferentes, dentro da mesma coluna e do mesmo fator (cultivar, dose de $\mathrm{P}$ no solo ou teor de $\mathrm{P}$ na semente), diferem pelo teste Tukey a $5 \%$. 
ao crescimento vegetal não pode ser atribuído apenas ao maior fornecimento deP, mas também ao estímulo ao crescimento inicial das plântulas. Na verdade, o aumento da área foliar em plantas oriundas de sementes com alto teor de P logo no início do ciclo (Quadro 3) pode provocar efeitos cumulativos nas taxas de crescimento posteriores, mesmo admitindo taxas de assimilação líquidas similares (De Marco, 1990).

\section{CONCLUSÕES}

1. Sementes com maior teor de P, obtidas de plantas que receberam adubação foliar com $\mathrm{P}$, podem aumentar o crescimento da parteaérea, a nodulação e a acumulação de $\mathrm{N}$ em plantas de feijoeiro no estádio vegetativo de crescimento, particularmente sob baixas doses de P aplicadas ao solo.

\section{LITERATURA CITADA}

ARAÚJ O, A.P. \& TEIXEIRA, M.G. Ontogenetic variations on absorption and utilization of phosphorus in common bean cultivars under biological nitrogen fixation. Plant Soil, 225:1-10, 2000.

ARAÚJ O, A.P.;TEIXEIRA, M.G. \& ALMEIDA, D.L. Growth and yield of common bean cultivars at two soil phosphorus levels under biological nitrogen fixation. Pesq. Agropec. Bras., 35:809-817, 2000.

BOLLAND, M.D.A. \& BAKER, M.J. High phosphorus concentrations in seed of wheat and annual medic are related to higher rates of dry matter production of seedlings and plants. Aust. J. Exp. Agric., 28:765-770, 1988.

BOLLAND, M.D.A. \& PAYNTER, B.H. Increasing phosphorus concentration in seed of annual pasture legume species increases herbage and seed yiel ds. Plant Soil, 125:197-205, 1990.

BURNETT, V.F.; COVENTRY, D.R. \& NEWTON, P.J . Effect of seed source and seed phosphorus content on the growth and yield of wheat in north-eastern Victoria. Aust. J. Exp. Agric., 37:191-198, 1997.

CASSMAN, K.G.; WHITNEY, A.S. \& STOCKINGER, K.R. Root growth and dry matter distribution of soybean as affected by phosphorus stress, nodulation, and nitrogen source. Crop Sci., 20:239-244, 1980.

CHAVERRA, M.H. \& GRAHAM, P.H. Cultivar variation in traits affecting early nodulation of common bean. Crop Sci., 32:1432-1436, 1992.

DE MARCO, D.G. Effect of seed weight, and seed phosphorus and nitrogen concentrations on the early growth of wheat seedlings. Aust. J. Exp. Agric., 30:545-549, 1990.
EMPRESA BRASILEIRA DE PESQUISA AGROPECUÁRIA EMBRAPA. CentroNacional de Pesquisa de Solos. Manual de métodos de análise de solo. 2.ed. Rio deJ aneiro, Centro Nacional de Pesquisa de Solos, 1997. 212p.

GATES, C.T. \& WILSON, J.R. The interaction of nitrogen and phosphorus on the growth, nutrient status and nodulation of Stylosanthes humilis H.B.K. (townsville stylo). Plant Soil, 41:325-333, 1974.

GRAHAM, P.H. \& ROSAS, J.C. Phosphorus fertilization and symbiotic nitrogen fixation in common bean. Agron. J., 71:925-926, 1979.

ISRAEL, D.W. Investigation of the role of phosphorus in symbiotic dinitrogen fixation. Plant Physiol., 84:835-840, 1987.

MALAVOLTA, E.; VITTI, G.C. \& OLIVEIRA, S.A. Avaliação do estado nutricional das plantas. Piracicaba, Associação Brasileira para Pesquisa da Potassa e do Fosfato, 1989. 201p.

NETER, J .; WASSERMAN, W. \& KUTNER, M.H. Applied linear statistical models. 3.ed. Burr Ridge, Richard D. Irwin, 1990. 1181p.

PAYNTER, B.H. Effect of external phosphorus and seed phosphorus supply on the shoot and root growth of yellow serradella, burr medic, and subterranean clover. J. Plant Nutr., 16:2313-2331, 1993.

PEREIRA, P.A.A. \& BLISS, F.A. Nitrogen fixation and plant growth of common bean (Phaseol us vulgaris L.) at different levels of phosphorus availability. Plant Soil, 104:79-84, 1987.

PIHA, M.I \& \& MUNNS, D.N . Nitrogen fixation potential of beans (Phaseol us vulgaris L.) compared with other grain legumes under controlled conditions. Plant Soil, 98:169-182, 1987.

SA, T.M. \& ISRAEL, D.W. Energy status and functioning of phosphorus-deficient soybean nodules. Plant Physiol., 97:928-935, 1991.

SESAY, A. \& SHIBLES, R. Mineral depletion and leaf senescence in soybean as influenced by foliar nutrient application during seed filling. Ann. Bot., 45:47-55, 1980.

TEIXEIRA, M.G. \& ARAÚJ O, A.P. Aumento do teor de fósforo em sementes de feijoeiro através da adubação foliar. In: REUNIÃO NACIONAL DE PESQUISA DE FEIJ ÃO, 6., Salvador, 1999. Resumos expandidos. Santo Antônio de Goiás, Embrapa Arroz e Feijão, 1999. p.756-759.

TEIXEIRA, M.G.; GUERRA, J.G.M.; ALMEIDA, D.L.; ARAÚJ O, A.P. \& FRANCO, A.A. Effect of seed phosphorus concentration on nodulation and growth of three common bean cultivars. J. Plant Nutr., 22:1599-1611, 1999.

THOMSON, B.D.; BELL, R.W. \& BOLLAND, M.D.A. Low seed phosphorus concentration depresses early growth and nodulation of narrow-leafed lupin (Lupinus angustifolius cv. Gungurru). J. Plant Nutr., 14:1355-1367, 1991.

VIEIRA, R.F. Desempenho de sementes de feijão provenientes de diferentes níveis de adubação. Pesq. Agropec. Bras., 21:1161-1168, 1986 
A.P. ARAÚj $O$ et al. 